\title{
NEW SUBCLASS OF ANALYTIC FUNCTIONS IN CONICAL DOMAIN ASSOCIATED WITH RUSCHEWEYH $q$-DIFFERENTIAL OPERATOR
}

\author{
SHAHID KHAN ${ }^{1, *}$, SAQIB HUSSAIN ${ }^{2}$, MUHAMMAD ASAD ZAIGHUM ${ }^{1}$, MUHAMMAD $^{2}$ \\ MUMTAZ KHAN ${ }^{3}$ \\ ${ }^{1}$ Department of Mathematics, Riphah International University Islamabad, Pakistan \\ ${ }^{2}$ Department of Mathematics, COMSATS Institute of Information Technology, Abbottabad, Pakistan \\ ${ }^{3}$ Department of Public Health Riphah University of Haripur, Pakistan \\ *Corresponding author: shahidmath761@gmail.com
}

\begin{abstract}
In this paper, we consider a new class of analytic functions which is defined by means of a Ruscheweyh q-differential operator. We investigated some new results such as coefficients inequalities and other interesting properties of this class. Comparison of new results with those that were obtained in earlier investigation are given as Corollaries.
\end{abstract}

\section{INTRODUCTION}

Let $\mathcal{A}$ denote the class of functions $f$ analytic in the open unit disk

$$
E=\{z: z \in \mathbb{C} \text { and }|z|<1\}
$$

and satisfying the normalization condition

$$
f(0)=0 \quad \text { and } \quad f^{\prime}(0)=1 .
$$

Received 2017-09-25; accepted 2017-12-07; published 2018-03-07.

2010 Mathematics Subject Classification. Primary 05A30, 30C45; Secondary 11B65, 47B38.

Key words and phrases. analytic functions; Ruscheweyh $q$-differential operator; $q$-derivative operator; conic domains.

(c)2018 Authors retain the copyrights of their papers, and all open access articles are distributed under the terms of the Creative Commons Attribution License. 
Thus, the functions in $\mathcal{A}$ are represented by the Taylor-Maclaurin series expansion given by

$$
f(z)=z+\sum_{n=2}^{\infty} a_{n} z^{n}, \quad(z \in E)
$$

Let $\mathcal{S}$ be the subset of $\mathcal{A}$ consisting of the functions that are univalent in $E$. Given functions $f, g \in \mathcal{A}, f$ is said to be subordinate to $g$ in $E$, denoted by

$$
f \prec g \quad \text { or } \quad f(z) \prec g(z) \quad(z \in E)
$$

if there exists a function $w \in \mathcal{P}_{0}$ where

$$
\mathcal{P}_{0}=\{w \in \mathcal{A}: w(0)=0, \quad \text { and }|w(z)|<1 \quad(z \in E)\},
$$

such that

$$
f(z)=g(w(z)) \quad(z \in E) .
$$

If $g$ is univalent in $E$, then it follows that

$$
f(z) \prec g(z) \quad(z \in E), \Rightarrow f(0)=0 \text { and } f(E) \subset g(E) .
$$

Kanas and Wiśniowska $[5,6]$ introduced the conic domain $\Omega_{k}, k \geq 0$ as

$$
\Omega_{k}=\left\{u+i v: u>k \sqrt{(u-1)^{2}+v^{2}}\right\} .
$$

We note that $\Omega_{k}$ is a region in the right half-plane, symmetric with respect to real axis, and contains the point $(1,0)$. More precisely for $k=0, \Omega_{0}$ is the right half-plane, for $0<k<1, \Omega_{k}$ is an unbounded region having boundary $\partial \Omega_{k}$, a rectangular hyperbola; for $k=1, \Omega_{1}$ is still an unbounded region where $\partial \Omega_{1}$ is a parabola, and for $k>1, \Omega_{k}$ is a bounded region enclosed by an ellipse. The extremal functions for these conic regions are

$$
p_{k}(z)= \begin{cases}\frac{1+z}{1-z}, & k=0, \\ 1+\frac{2}{\pi^{2}}\left(\log \frac{1+\sqrt{z}}{1-\sqrt{z}}\right)^{2}, & k=1, \\ \frac{1}{1-k^{2}} \cosh \left\{\left(\frac{2}{\pi} \arccos k\right) \log \frac{1+\sqrt{z}}{1-\sqrt{z}}\right\}-\frac{k^{2}}{1-k^{2}}, & 0<k<1, \\ \frac{1}{k^{2}-1} \sin \left(\frac{\pi}{2 K(\kappa)} \int_{0}^{\frac{u(z)}{\sqrt{\kappa}}} \frac{d t}{\sqrt{1-t^{2}} \sqrt{1-\kappa^{2} t^{2}}}\right)+\frac{k^{2}}{k^{2}-1}, & k>1,\end{cases}
$$

where

$$
u(z)=\frac{z-\sqrt{\kappa}}{1-\sqrt{\kappa} z} \quad(z \in E)
$$

and $\kappa \in(0,1)$ is chosen such that $k=\cosh \left(\pi K^{\prime}(\kappa) /(4 K(\kappa))\right)$. Here $K(\kappa)$ is Legender's complete elliptic integral of first kind and $K^{\prime}(\kappa)=K\left(\sqrt{1-\kappa^{2}}\right)$ and $K^{\prime}(t)$ is the complementary integral of $K(t)$ for details 
see $[1,5,6]$ and more recently $[9,12,14]$. If $p_{k}(z)=1+L_{1}(k) z+L_{2}(k) z^{2}+\ldots, z \in E$. Then it was shown in [6] that for $(1.2)$ one can have

$$
\begin{array}{ll}
L_{1}(k)= \begin{cases}\frac{2 A^{2}}{1-k^{2}}, & 0 \leq k<1 \\
\frac{8}{\pi^{2}}, & k=1, \\
\frac{\pi^{2}}{4 K^{2}(t)^{2}(1+t) \sqrt{t}}, & k>1,\end{cases} \\
L_{2}(k)=D(k) L_{1}(k),
\end{array}
$$

where

$$
D(k)= \begin{cases}\frac{A^{2}+2}{3}, & 0 \leq k<1 \\ \frac{8}{\pi^{2}}, & k=1 \\ \frac{(4 K(t))^{2}\left(t^{2}+6 t+1\right)-\pi^{2}}{24 K(t)^{2}(1+t) \sqrt{t}}, & k>1\end{cases}
$$

with $A=\frac{2}{\pi} \arccos k$.

Furthermore a function $p$ is said to be in the class $k-P[A, B]$, if and only if

$$
p(z) \prec \frac{(A+1) p_{k}(z)-(A-1)}{(B+1) p_{k}(z)-(B-1)}, \quad k \geq 0,
$$

where $p_{k}$ is defined in (1.2) and $-1 \leq B<A \leq 1$. Geometrically, the function $p \in k-P[A, B]$ takes all values from the domain $\Omega_{k}[A, B],-1 \leq B<A \leq 1, k \geq 0$ which is defined as:

$$
\Omega_{k}[A, B]=\left\{w: \Re\left(\frac{(B-1) w-(A-1)}{(B+1) w-(A+1)}\right)>k\left|\frac{(B-1) w-(A-1)}{(B+1) w-(A+1)}-1\right|\right\},
$$

or equivalently $\Omega_{k}[A, B]$ is a set of numbers $w=u+i v$ such that

$$
\begin{aligned}
& {\left[\left(B^{2}-1\right)\left(u^{2}+v^{2}\right)-2(A B-1) u+\left(A^{2}-1\right)\right]^{2} } \\
> & k\left[\left(-2(B+1)\left(u^{2}+v^{2}\right)+2(A+B+2) u-2(A+1)\right)^{2}+4(A-B)^{2} v^{2}\right] .
\end{aligned}
$$

This domain represents the conic type regains for detail see [11]. It can be easily seen that $0-P[A, B]=$ $P[A, B]$ introduced in [4] and $k-P[1,-1]=P\left(p_{k}\right)$ introduced in [5].

We now recall some basic concept details of the $q$-calculus which are used in this paper. Throughout this paper we assume $q$ to be a fixed number between 0 and 1 .

For any non-negative integer $n$, the $q$-integer number $n,[n, q]$ is defined by:

$$
[n, q]=\frac{1-q^{n}}{1-q}=1+q+\ldots+q^{n-1}, \quad[0, q]=0
$$

The $q$-number shifted factorial is defined by $[0, q] !=1$ and $[n, q] !=[1, q][2, q] \ldots[n, q]$. Clearly, $\lim _{q \rightarrow 1}[n, q]=n$ and $\lim _{q \rightarrow 1}[n, q] !=n !$. In general we will denote $[t, q]=\frac{1-q^{t}}{1-q}$ also for a non-integer number. Let $f \in \mathcal{A}$, and let the $q$-derivative operator or $q$-difference operator be defined by

$$
\partial_{q} f(z)=\frac{f(q z)-f(z)}{(q-1) z} \quad(z \in E)
$$


It is easy to observed that for $n \in \mathbb{N}:=\{1,2,3, \ldots\}$ and $z \in E$

$$
\partial_{q} z^{n}=[n, q] z^{n-1}
$$

Let the $q$-generalized Pochhammer symbol be defined as

$$
[t, q]_{n}=[t, q][t+1, q][t+2, q] \ldots[t+n-1, q],
$$

and for $t>0$ let the $q$-gamma function be defined as

$$
\Gamma_{q}(t+1)=[t] \Gamma_{q}(t) \quad \text { and } \quad \Gamma_{q}(1)=1 .
$$

The study of opretors play in important role in Geomatric Functions Theory. Several diffierential and integral operators were introduced and studied, see for example $[2,3,13]$. Kannas et al. define Ruscheweyh $q$-differential operator as follow:

Definition 1.1. [7] For $f \in \mathcal{A}$, let the Ruscheweyh q-differential operator be defined as follows:

$$
R_{q}^{\lambda} f(z)=f(z) * F_{q, \lambda+1}(z), \quad(z \in E, \lambda>-1)
$$

where

$$
\begin{aligned}
F_{q, \lambda+1}(z) & =z+\sum_{n=2}^{\infty} \frac{\Gamma_{q}(n+\lambda)}{[n-1, q] ! \Gamma_{q}(1+\lambda)} z^{n} \\
& =z+\sum_{n=2}^{\infty} \frac{[\lambda+1, q]_{n-1}}{[n-1, q] !} z^{n} \\
& =z+\sum_{n=2}^{\infty} \varphi_{n-1} z^{n}
\end{aligned}
$$

Where

$$
\varphi_{n-1}=\frac{\Gamma_{q}(n+\lambda)}{[n-1, q] ! \Gamma_{q}(1+\lambda)}=\frac{[\lambda+1, q]_{n-1}}{[n-1, q] !}
$$

From (1.6) we obtain that

$$
R_{q}^{0} f(z)=f(z), \quad R_{q}^{1} f(z)=z \partial_{q} f(z)
$$

and

$$
R_{q}^{m} f(z)=\frac{z \partial_{q}^{m}\left(z^{m-1} f(z)\right)}{[m, q] !}, \quad(m \in N)
$$

Making use of (1.6) and (1.7), the power series of $R_{q}^{\lambda} f(z)$ is given by

$$
R_{q}^{\lambda} f(z)=z+\sum_{n=2}^{\infty} \frac{\Gamma_{q}(n+\lambda)}{[n-1, q] ! \Gamma_{q}(1+\lambda)} a_{n} z^{n}=z+\sum_{n=2}^{\infty} \frac{[\lambda+1, q]_{n-1}}{[n-1, q] !} a_{n} z^{n} .
$$

Note that

$$
\lim _{q \rightarrow 1} F_{q, \lambda+1}(z)=\frac{z}{(1-z)^{\lambda+1}}
$$


and

$$
\lim _{q \rightarrow 1} R_{q}^{\lambda} f(z)=f(z) * \frac{z}{(1-z)^{\lambda+1}}
$$

Thus, we can say that Ruscheweyh $q$-differential operator reduces to the differential operator defined by Ruscheweyh [16] in the case when $q \rightarrow 1$. It is easy to check that

$$
z \partial\left(F_{q, \lambda+1}(z)\right)=\left(1+\frac{[\lambda, q]}{q^{\lambda}}\right) F_{q, \lambda+2}(z)-\frac{[\lambda, q]}{q^{\lambda}} F_{q, \lambda+1}(z) .
$$

Making use of (1.6), (1.9) and the properties of Hadamard product, we obtain the following equality

$$
z \partial\left(R_{q}^{\lambda} f(z)\right)=\left(1+\frac{[\lambda, q]}{q^{\lambda}}\right) R_{q}^{\lambda+1} f(z)-\frac{[\lambda, q]}{q^{\lambda}} R_{q}^{\lambda} f(z) .
$$

If $q \rightarrow 1$, the equality (1.10) implies

$$
z\left(R^{\lambda} f(z)\right)^{\prime}=(1+\lambda) R^{\lambda+1} f(z)-\lambda R^{\lambda} f(z)
$$

which is the well known recurrent formula for Ruscheweyh differential operator.

Using Ruscheweyh differential operator various new classes of convex and starlike functions have been defined. Now by using Ruscheweyh $q$-differential operator we introduce the following class of functions.

Definition 1.2. A function $f(z) \in A$ is said to be in the class $k-\mathcal{U} \mathcal{S}_{q}(\lambda, A, B, \beta), k \geq 0,-1 \leq B<A \leq 1$, if and only if

$$
\Re\left(\frac{(B-1) G(z)-(A-1)}{(B+1) G(z)-(A+1)}\right)>k\left|\frac{(B-1) G(z)-(A-1)}{(B+1) G(z)-(A+1)}-1\right|
$$

where

$$
G(z)=\frac{z \partial_{q} R_{q}^{\lambda} f(z)}{R_{q}^{\lambda} f(z)}+\beta \frac{z^{2} \partial_{q}^{2} R_{q}^{\lambda} f(z)}{R_{q}^{\lambda} f(z)},
$$

or equivalently

$$
\frac{z \partial_{q} R_{q}^{\lambda} f(z)}{R_{q}^{\lambda} f(z)}+\beta \frac{z^{2} \partial_{q}^{2} R_{q}^{\lambda} f(z)}{R_{q}^{\lambda} f(z)} \in k-P[A, B] .
$$

Remark 1.1. It is easily see that

$$
\lim _{q \rightarrow 1^{-}} k-\mathcal{U} \mathcal{S}_{q}(0, A, B, 0)=k-\mathcal{S T}(A, B)
$$

where $k-\mathcal{S T}(A, B)$ is a functions class, intrioduced and studied by Noor and Sarfraz [11].

Each of the following lemmas will be needed in our present investigation.

Lemma 1.1. [15] Let $h(z)=1+\sum_{n=1}^{\infty} c_{n} z^{n}$ be subordinate to $H(z)=1+\sum_{n=1}^{\infty} C_{n} z^{n}$. If $H(z)$ is univalent in $E$ and $H(E)$ is convex, then

$$
\left|c_{n}\right| \leq\left|C_{1}\right|, \quad n \geq 1
$$


Lemma 1.2. ( [8], [10]) If $q(z)=1+c_{1} z+c_{2} z^{2}+\ldots$ is an analytic function with positive real part in $E$, then

$$
\left|c_{2}-v c_{1}^{2}\right| \leq 2 \max \{1,|2 v-1|\}
$$

The result is sharp for the functions

$$
q(z)=\frac{1+z^{2}}{1-z^{2}}, \text { or } q(z)=\frac{1+z}{1-z}
$$

Lemma 1.3. [8] Let the function $w \in E$ be given by

$$
w(z)=c_{1} z+c_{2} z^{2}+\ldots \quad z \in E
$$

Then for every complex number $v$,

$$
\left|c_{2}-v c_{1}^{2}\right| \leq 1+(|v|-1)\left|c_{1}\right|^{2} .
$$

Lemma 1.4. [11] Let $k \in[0, \infty)$ be a fixed and

$$
q_{k}(z)=\frac{(A+1) p_{k}(z)-(A-1)}{(B+1) p_{k}(z)-(B-1)}
$$

then

$$
q_{k}(z)=1+H_{1}(k) z+H_{2}(k) z^{2}+\ldots, z \in E .
$$

and

$$
\begin{aligned}
& H_{1}:=H_{1}(k)=\frac{A-B}{2} L_{1}(k), \\
& H_{2}:=H_{2}(k)=\frac{A-B}{4}\left\{2 D(k)-(B+1) H_{1}\right\} L_{1}(k)
\end{aligned}
$$

where $L_{1}(k)$ and $D(k)$ are defined in (1.3) and (1.4).

\section{Main Results}

Theorem 2.1. A function $f \in \mathcal{A}$ and of the form (1.1) is in the class $k-\mathcal{U} \mathcal{S}_{q}(\lambda, A, B, \beta)$, if it satisfies the condition

$$
\sum_{n=2}^{\infty}\left\{\begin{array}{c}
\{2(k+1)\{1-[n, q]-\beta[n, q][n-1, q]\} \\
+|\{(B+1)[n, q]+\beta[n, q][n-1, q]-(A+1)\}|\}
\end{array}\right\} \varphi_{n-1}\left|a_{n}\right| \leq|B-A|
$$

where $-1 \leq B<A \leq 1, \beta \geq 0$ and $k \geq 0$. 
Proof. Assume (2.1) is hold, then it suffices to show that

$$
\left\{\begin{array}{r}
k\left|\frac{(B-1)\left(\frac{z \partial_{q} R_{f}^{\lambda} f(z)}{R_{q}^{\lambda} f(z)}+\beta \frac{z^{2} \partial_{q}^{2} R_{q}^{\lambda} f(z)}{R_{q}^{\lambda} f(z)}\right)-(A-1)}{(B+1)\left(\frac{z \partial_{q}^{2} R_{f}^{\lambda} f(z)}{R_{q}^{\lambda} f(z)}+\beta \frac{z_{q}^{2} \partial_{q}^{2} R_{q}^{\lambda} f(z)}{R_{q}^{\lambda} f(z)}\right)-(A+1)}-1\right| \\
-\Re\left\{\frac{(B-1)\left(\frac{z \partial_{q} R_{q}^{\lambda} f(z)}{R_{q}^{\lambda} f(z)}+\beta \frac{z^{2} \partial_{q}^{2} R_{q}^{\lambda} f(z)}{R_{q}^{\lambda} f(z)}\right)-(A-1)}{(B+1)\left(\frac{z \partial_{q} R_{q}^{\lambda} f(z)}{R_{q}^{\lambda} f(z)}+\beta \frac{z_{q}^{2} \partial_{q}^{2} R_{q}^{\lambda} f(z)}{R_{q}^{\lambda} f(z)}\right)-(A+1)}-1\right\}
\end{array}\right\}<1,
$$

We have

$$
\begin{aligned}
& \left\{\begin{array}{r}
k\left|\frac{(B-1)\left(\frac{z \partial_{q} R_{q}^{\lambda} f(z)}{R_{q}^{\lambda}(z)}+\beta \frac{z^{2} \partial_{q}^{2} R_{q}^{\lambda} f(z)}{R_{q}^{\lambda} f(z)}\right)-(A-1)}{(B+1)\left(\frac{z \partial_{q} R_{q}^{\lambda} f(z)}{R_{q}^{\lambda} f(z)}+\beta \frac{z^{2} \partial_{q}^{2} R_{q}^{\lambda} f(z)}{R_{q}^{\lambda} f(z)}\right)-(A+1)}-1\right| \\
-\Re\left\{\frac{(B-1)\left(\frac{z \partial_{q} R_{q}^{\lambda} f(z)}{R_{q}^{\lambda} f(z)}+\beta \frac{z^{2} \partial_{q}^{2} R_{q}^{\lambda} f(z)}{R_{q}^{\lambda} f(z)}\right)-(A-1)}{(B+1)\left(\frac{z \partial_{q} R_{q}^{\lambda} f(z)}{R_{q}^{\lambda} f(z)}+\beta \frac{z^{2} \partial_{q}^{2} R_{q}^{\lambda} f(z)}{R_{q}^{\lambda} f(z)}\right)-(A+1)}-1\right\}
\end{array}\right\} \\
& \leq(k+1)\left|\frac{(B-1)\left(z \partial_{q} R_{q}^{\lambda} f(z)+\beta z^{2} \partial_{q}^{2} R_{q}^{\lambda} f(z)\right)-(A-1) R_{q}^{\lambda} f(z)}{(B+1)\left(z \partial_{q} R_{q}^{\lambda} f(z)+\beta z^{2} \partial_{q}^{2} R_{q}^{\lambda} f(z)\right)-(A+1) R_{q}^{\lambda} f(z)}-1\right| \\
& =2(k+1)\left|\frac{R_{q}^{\lambda} f(z)-z \partial_{q} R_{q}^{\lambda} f(z)-\beta z^{2} \partial_{q}^{2} R_{q}^{\lambda} f(z)}{(B+1)\left(z \partial_{q} R_{q}^{\lambda} f(z)+\beta z^{2} \partial_{q}^{2} R_{q}^{\lambda} f(z)\right)-(A+1) R_{q}^{\lambda} f(z)}\right| \\
& =2(k+1) \frac{\sum_{n=2}^{\infty}(1-[n, q]-\beta[n, q][n-1, q]) \varphi_{n-1} a_{n} z^{n}}{(B-A) z+\sum_{n=2}^{\infty}\left\{(B+1)[n, q]_{q}+\beta[n, q][n-1, q]-(A+1)\right\} \varphi_{n-1} a_{n} z^{n}} \\
& \leq 2(k+1) \frac{\sum_{n=2}^{\infty}(1-[n, q]-\beta[n, q][n-1, q]) \varphi_{n-1}\left|a_{n}\right|}{|B-A|-\sum_{n=2}^{\infty}\left\{(B+1)[n, q]_{q}+\beta[n, q][n-1, q]-(A+1)\right\} \varphi_{n-1}\left|a_{n}\right|} \\
& <1 \quad \text { (by }(2.1)) \text {. }
\end{aligned}
$$

When $A=1-2 \alpha, B=-1, \beta=0$ with $0 \leq \alpha<1$, then we have the following known result, proved by Kanas and Raducanu in [7].

Corollary 2.1. A function $f \in A$ and of the form (1.1) is in the class $k-\mathcal{U S} \mathcal{S}_{q}(\lambda, 1-2 \alpha,-1)$, if it satisfies the condition

$$
\sum_{n=2}^{\infty}\{(k+1)[n, q]-k-\alpha\} \varphi_{n-1}\left|a_{n}\right| \leq 1-\alpha .
$$

When $q \rightarrow 1, \beta=0, \lambda=0$, then we have the following known result, proved by Noor and Sarfraz [11].

Corollary 2.2. A function $f \in A$ and of the form (1.1 is in the class $k-\mathcal{S T}(A, B)$, if it satisfies the condition

$$
\sum_{n=2}^{\infty}\{2(k+1)(n-1)+|n(B+1)-(A+1)|\}\left|a_{n}\right| \leq|B-A| .
$$


When $q \rightarrow 1, \lambda=0, \beta=0, A=1-2 \alpha, B=-1$ with $0 \leq \alpha<1$, then we have the following known result, proved by Shams et-al. in [18].

Corollary 2.3. A function $f \in A$ and of the form (1.1) is in the class $k-\mathcal{U S T}(1-2 \alpha,-1)$, if it satisfies the condition

$$
\sum_{n=2}^{\infty}\{n(k+1)-(k+\alpha)\}\left|a_{n}\right| \leq 1-\alpha
$$

where $0 \leq \alpha<1$ and $k \geq 0$.

When $\lambda=0, \beta=0, A=1-2 \alpha, B=-1$ with $0 \leq \alpha<1$ and $k=0$, then we have the following known result, proved by Selverman in [17].

Corollary 2.4. A function $f \in A$ and of the form (1.1) is in the class $0-\mathcal{U S T}(1-2 \alpha,-1)$, if it satisfies the condition

$$
\sum_{n=2}^{\infty}\{n-\alpha\}\left|a_{n}\right| \leq 1-\alpha, \quad 0 \leq \alpha<1
$$

Theorem 2.2. If $f(z) \in k-\mathcal{U S}_{q}(\lambda, A, B, \beta)$ and is of the form (1.1). Then

$$
\left|a_{n}\right| \leq \prod_{j=0}^{n-2}\left(\frac{\left|L_{1}(k)(A-B)-2[j, q] B\right|}{2[j+1, q]\{q+\beta[j+2, q]\} \varphi_{j+1}}\right), \quad n \geq 2,
$$

where $L_{1}(k)$ is defined by (1.3).

Proof. Let

$$
\frac{z \partial_{q} R_{q}^{\lambda} f(z)}{R_{q}^{\lambda} f(z)}+\beta \frac{z^{2} \partial_{q}^{2} R_{q}^{\lambda} f(z)}{R_{q}^{\lambda} f(z)}=p(z)
$$

Then

$$
\begin{aligned}
p(z) \prec & \frac{(A+1) p_{k}(z)-(A-1)}{(B+1) p_{k}(z)-(B-1)} \\
= & {\left[(A+1) p_{k}(z)-(A-1)\right]\left[(B+1) p_{k}(z)-(B-1)\right]^{-1} } \\
= & \frac{(A-1)}{(B-1)}\left[1-\frac{(A+1)}{(A-1)} p_{k}(z)\right]\left[1+\sum\left(\frac{(B+1)}{(B-1)} p_{k}(z)\right)^{n}\right] \\
= & \frac{(A-1)}{(B-1)}+\left(\frac{(A-1)(B+1)}{(B-1)^{2}}-\frac{(A+1)}{(B-1)}\right)\left(p_{k}(z)\right) \\
& +\left(\frac{(A-1)(B+1)^{2}}{(B-1)^{3}}-\frac{(A+1)(B+1)}{(B-1)^{2}}\right)\left(p_{k}(z)\right)^{2}+\ldots
\end{aligned}
$$

By taking

$$
p_{k}(z)=1+L_{1}(k) z+L_{2}(k) z^{2}+\ldots
$$

after some simplification, we obtain

$$
p(z) \prec \sum_{n=1}^{\infty} \frac{-2(B+1)^{n-1}}{(B-1)^{n}}+\left\{\sum_{n=1}^{\infty} \frac{-2 n(A-B)(B+1)^{n-1}}{(B-1)^{n+1}}\right\} L_{1}(k)+\ldots
$$


Now we see that the series $\sum_{n=1}^{\infty} \frac{-2(B+1)^{n-1}}{(B-1)^{n}}$ and $\sum_{n=1}^{\infty} \frac{-2 n(A-B)(B+1)^{n-1}}{(B-1)^{n+1}}$ are convergent and converge to 1 and $\frac{A-B}{2}$ respectively. Therefore,

$$
p(z) \prec 1+\frac{A-B}{2} L_{1}(k) z+\ldots
$$

Now if $p(z)=1+\sum_{n=1}^{\infty} c_{n} z^{n}$, then by Lemma 1 , we have

$$
\left|c_{n}\right| \leq \frac{A-B}{2} L_{1}(k), \quad n \geq 1
$$

Now from (2.3), we have

$$
z \partial_{q} R_{q}^{\lambda} f(z)+\beta z^{2} \partial_{q}^{2} R_{q}^{\lambda} f(z)=R_{q}^{\lambda} f(z) p(z)
$$

which implies that

$$
z+\sum_{n=2}^{\infty}\{[n, q]+\beta[n, q][n-1, q]\} \varphi_{n-1} a_{n} z^{n}=\left(1+\sum_{n=1}^{\infty} c_{n} z^{n}\right)\left(z+\sum_{n=2}^{\infty} \varphi_{n-1} a_{n} z^{n}\right) .
$$

Equating coefficients of $z^{n}$ on both sides, we have

$$
[n-1, q]\{q+\beta[n, q]\} \varphi_{n-1} a_{n}=\sum_{j=1}^{n-1} \varphi_{j-1} a_{j} c_{n-j}, \quad a_{1}=1 .
$$

This implies that

$$
\left|a_{n}\right| \leq \frac{1}{[n-1, q]\{q+\beta[n, q]\} \varphi_{n-1}} \sum_{j=1}^{n-1} \varphi_{j-1}\left|a_{j}\right|\left|c_{n-j}\right|, \quad a_{1}=1 .
$$

Using (2.4), we have

$$
\left|a_{n}\right| \leq \frac{(A-B)\left|L_{1}(k)\right|}{2[n-1, q]\{q+\beta[n, q]\} \varphi_{n-1}} \sum_{j=1}^{n-1} \varphi_{j-1}\left|a_{j}\right|, \quad a_{1}=1 .
$$

Now we prove that

$$
\frac{(A-B)\left|L_{1}(k)\right|}{2[n-1, q]\{q+\beta[n, q]\} \varphi_{n-1}} \sum_{j=1}^{n-1} \varphi_{j-1}\left|a_{j}\right| \leq \prod_{j=0}^{n-2}\left(\frac{\left|L_{1}(k)(A-B)-2[j, q] B\right|}{2[j+1, q]\{q+\beta[j+2, q]\}}\right) .
$$

For this we use the induction method

For $n=2$, from (2.5), we have

$$
\left|a_{2}\right| \leq \frac{(A-B)\left|L_{1}(k)\right|}{2\{q+\beta[2, q]\} \varphi_{1}}
$$

From (2.2), we have

$$
\left|a_{2}\right| \leq \frac{(A-B)\left|L_{1}(k)\right|}{2\{q+\beta[2, q]\} \varphi_{1}} .
$$

For $\mathrm{n}=3$ from $(2.5)$, we have

$$
\begin{aligned}
\left|a_{3}\right| & \leq \frac{(A-B)\left|L_{1}(k)\right|}{2[2, q]\{q+\beta[3, q]\} \varphi_{2}}\left\{1+\varphi_{1} a_{2}\right\} \\
& \leq \frac{(A-B)\left|L_{1}(k)\right|}{2[2, q]\{q+\beta[3, q]\} \varphi_{2}}\left\{1+\frac{(A-B)\left|L_{1}(k)\right|}{2\{q+\beta[2, q]\}}\right\} .
\end{aligned}
$$


From (2.2), we have

$$
\begin{aligned}
\left|a_{3}\right| & \leq \frac{(A-B)\left|L_{1}(k)\right|}{2\{q+\beta[2, q]\} \varphi_{1}}\left\{\left(\frac{\left|(A-B) L_{1}(k)-2 B\right|}{2[2, q]\{q+\beta[3, q]\} \varphi_{2}}\right)\right\} \\
& \leq \frac{(A-B)\left|L_{1}(k)\right|}{2\{q+\beta[2, q]\} \varphi_{1}}\left\{\left(\frac{(A-B)\left|L_{1}(k)\right|+2|B|}{2[2, q]\{q+\beta[3, q]\} \varphi_{2}}\right)\right\} \\
& \leq \frac{(A-B)\left|L_{1}(k)\right|}{2[2, q]\{q+\beta[3, q]\} \varphi_{2}}\left\{\frac{(A-B)\left|L_{1}(k)\right|}{2\{q+\beta[2, q]\} \varphi_{1}}+\frac{1}{\{q+\beta[2, q]\} \varphi_{1}}\right\} .
\end{aligned}
$$

Let the hypothesis be true for $n=m$. From (2.4), we have

$$
\left|a_{m}\right| \leq \frac{(A-B)\left|L_{1}(k)\right|}{2[m-1, q]\{q+\beta[m, q]\} \varphi_{m-1}} \sum_{j=1}^{n-1}\left|a_{j}\right|, a_{1}=1
$$

From (2.2), we have

$$
\begin{aligned}
\left|a_{m}\right| & \leq \prod_{j=0}^{m-2}\left(\frac{\left|L_{1}(k)(A-B)-2[j, q] B\right|}{2[j+1, q]\{q+\beta[j+2, q]\} \varphi_{j+1}}\right), & & n \geq 2 \\
& \leq \prod_{j=0}^{m-2}\left(\frac{\left|L_{1}(k)\right|(A-B)+2[j, q]}{2[j+1, q]\{q+\beta[j+2, q]\} \varphi_{j+1}}\right), & & n \geq 2 .
\end{aligned}
$$

By the induction hypothesis, we have

$$
\frac{(A-B)\left|L_{1}(k)\right|}{2[m-1, q]\{q+\beta[m, q]\} \varphi_{m-1}} \sum_{j=1}^{m-1} \varphi_{j-1}\left|a_{j}\right| \leq \prod_{j=0}^{m-2}\left(\frac{\left|L_{1}(k)\right|(A-B)+2[j, q]}{2[j+1, q]\{q+\beta[j+2, q]\} \varphi_{j+1}}\right) .
$$

Multiplying both sides by (2.7)

$$
\frac{(A-B)\left|L_{1}(k)\right|+2[m-1, q]\{q+\beta[m, q]\}}{2[m-1, q]\{q+\beta[m, q]\} \varphi_{m-1}},
$$

we have

$$
\begin{aligned}
& \prod_{j=0}^{m-2}\left(\frac{\left|L_{1}(k)\right|(A-B)+2[j, q]}{2[j+1, q]\{q+\beta[j+1, q]\} \varphi_{j+1}}\right) \\
\geq & \left\{\frac{(A-B)\left|L_{1}(k)\right|+2[m-1, q]\{q+\beta[m, q]\}}{2[m-1, q]\{q+\beta[m, q]\} \varphi_{m-1}}\right\} \frac{(A-B)\left|L_{1}(k)\right|}{2[m-1, q]\{q+\beta[m, q]\} \varphi_{m-1}} \sum_{j=1}^{m-1} \varphi_{j-1}\left|a_{j}\right| \\
= & \frac{(A-B)\left|L_{1}(k)\right|}{2[m-1, q]\{q+\beta[m, q]\} \varphi_{m-1}}\left[\left\{\frac{(A-B)\left|L_{1}(k)\right|+2[m-1, q]\{q+\beta[m, q]\}}{2[m-1, q]\{q+\beta[m, q]\} \varphi_{m-1}} \sum_{j=1}^{m-1} \varphi_{j-1}\left|a_{j}\right|\right\}\right] \\
\geq & \frac{(A-B)\left|L_{1}(k)\right|}{2[m-1, q]\{q+\beta[m, q]\} \varphi_{m-1}}\left\{\left|a_{m=1}^{m-1} \varphi_{j-1}\right| a_{j} \mid\right. \\
= & \left.\frac{(A-B)\left|L_{1}(k)\right|}{2[m-1, q]\{q+\beta[m, q]\} \varphi_{m-1}} \sum_{j=1}^{m-1} \varphi_{j-1}\left|a_{j}\right|\right\}
\end{aligned}
$$


That is,

$$
\frac{(A-B)\left|L_{1}(k)\right|}{2[m-1, q]\{q+\beta[m, q]\} \varphi_{m-1}} \sum_{j=1}^{m} \varphi_{j-1}\left|a_{j}\right| \leq \prod_{j=0}^{m-2}\left(\frac{\left|L_{1}(k)\right|(A-B)+2[j, q]}{2[j+1, q]\{q+\beta[j+1, q]\} \varphi_{j+1}}\right) .
$$

which shows that inequality (2.7) is true for $n=m+1$. Hence the required result.

When $q \rightarrow 1, \lambda=0$ and $\beta=0$, then we have the following known result, proved by Noor and Sarfraz in [11].

Corollary 2.5. A function $f \in A$ and of the form (1.1) is in the class $k-\mathcal{S T}[A, B]$, if it satisfies the condition

$$
\left|a_{n}\right| \leq \prod_{j=0}^{n-2}\left(\frac{\left|L_{1}(k)(A-B)-2 j B\right|}{2(j+1)}\right) .
$$

When $\lambda=0, A=1, B=-1$ and $\beta=0$ then we have the following known result, proved by Kanas and Wisniowska in [6].

Corollary 2.6. A function $f \in A$ and of the form (1.1) is in the class $k-\mathcal{U S T}[A, B]$, if it satisfies the condition

$$
\left|a_{n}\right| \leq \prod_{j=0}^{n-2}\left(\frac{\left|L_{1}(k)+j\right|}{(j+1)}\right) .
$$

When $\lambda=0, A=1-2 \alpha, \beta=0, B=-1$ with $0 \leq \alpha<1$, then we have the following known result, proved by Shams et al. in [18].

Corollary 2.7. A function $f \in A$ and of the form (1.1) is in the class $\mathcal{S D}(k, \alpha)$, if it satisfies the condition

$$
\left|a_{n}\right| \leq \prod_{j=0}^{n-2}\left(\frac{\left|L_{1}(k)(1-\alpha)+j\right|}{(j+1)}\right) .
$$

where $0 \leq \alpha<1$ and $k \geq 0$.

When $\lambda=0, \beta=0, k=0$, then $T_{1}(k)=2$ and we get the following known result, proved in [4]

Corollary 2.8. A function $f \in A$ and of the form (1.1) is in the class $\mathcal{S}^{*}[A, B]$, if it satisfies the condition

$$
\left|a_{n}\right| \leq \prod_{j=0}^{n-2}\left(\frac{|(A-B)-j B|}{(j+1)}\right), \quad-1 \leq B<A \leq 1 .
$$

When $\lambda=0, \beta=0, A=1-2 \alpha, B=-1$ with $0 \leq \alpha<1$ and $k=0$, then we have the following known result, proved by Selverman in [17].

Corollary 2.9. A function $f \in A$ and of the form (1.1) is in the class $\mathcal{S}^{*}(\alpha)$, if it satisfies the condition

$$
\left|a_{n}\right| \leq \frac{\prod_{j=0}^{n-2}(j-2 \alpha)}{(n-1) !}, 0 \leq \alpha<1 .
$$


Theorem 2.3. Let $-1 \leq B<A \leq 1$ and $0 \leq k<\infty$ be fixed and let $f(z) \in k-\mathcal{U S} \mathcal{S}_{q}(\lambda, A, B, \beta)$ and is of the form (1.1) Then for a complex number $\mu$.

$$
\left|a_{3}-\mu a_{2}^{2}\right| \leq\left\{\begin{aligned}
\frac{(A-B) L_{1}(k)}{2[2, q]\{q+[3, q] \beta\} \varphi_{2}} \mid\left\{2+\frac{2 D(k)-(1+B) L_{1}(k)}{2}\left[2+\frac{2 D(k)-(1+B) L_{1}(k)}{2}\right.\right. & \left(\mu>\delta_{1}\right), \\
\left.\left.-\frac{(A-B)}{2\{q+\beta[2, q]\}} L_{1}(k)\left(1-\mu \frac{\varphi_{2}}{\left(\varphi_{1}\right)^{2}}\right)\right]\right\} \mid, & \left(\delta_{1} \leq \mu \leq \delta_{2}\right), \\
\frac{(A-B) L_{1}(k)}{2[2, q]\{q+[3, q] \beta\} \varphi_{2}} . & \left(\mu<\delta_{2}\right) . \\
\frac{(A-B) L_{1}(k)}{2[2, q]\{q+[3, q] \beta\} \varphi_{2}}\left[\frac{2 D(k)-(1+B) L_{1}(k)}{2}\right. & \\
\left.\frac{(A-B)}{2\{q+\beta[2, q]\}} L_{1}(k)\left(1-\mu \frac{\varphi_{2}}{\left(\varphi_{1}\right)^{2}}\right)\right] &
\end{aligned}\right.
$$

Where

$$
\begin{gathered}
\delta_{1}=\frac{\left(\varphi_{1}\right)^{2}}{\varphi_{2}(A-B) L_{1}(k)}\left[\begin{array}{c}
(q+\beta[2, q])\left\{2+2 D(k)-(1+B) L_{1}(k)\right\} \\
+(A-B) L_{1}(k)
\end{array}\right], \\
\delta_{2}=\frac{\left(\varphi_{1}\right)^{2}}{\varphi_{2}(A-B) L_{1}(k)}\left[\begin{array}{c}
(q+\beta[2, q])\left\{2 D(k)-(1+B) L_{1}(k)-2\right\} \\
+(A-B) L_{1}(k)
\end{array}\right] .
\end{gathered}
$$

and $L_{1}(k), D(k)$ are defined in (1.3) and (1.4).

Proof. If $f(z) \in k-\mathcal{U S}_{q}(\lambda, A, B, \beta)$ then it follows that

$$
\frac{z \partial_{q} R_{q}^{\lambda} f(z)}{R_{q}^{\lambda} f(z)}+\beta \frac{z^{2} \partial_{q}^{2} R_{q}^{\lambda} f(z)}{R_{q}^{\lambda} f(z)} \prec q_{k}(z)=1+\frac{A-B}{2} L_{1}(k) z+\frac{\left[2 D(k)-(1+B) L_{1}(k)\right](A-B)}{4} L_{1}(k) z^{2}+\ldots
$$

Now by the definition of subordination there exists a function $w$ analytic in $E$ with $w(0)=0$ and $|w(z)|<1$ such that

$$
\frac{z \partial_{q} R_{q}^{\lambda} f(z)}{R_{q}^{\lambda} f(z)}+\beta \frac{z^{2} \partial_{q}^{2} R_{q}^{\lambda} f(z)}{R_{q}^{\lambda} f(z)}=1+\frac{A-B}{2} L_{1}(k) w(z)+\frac{\left[2 D(k)-(1+B) L_{1}(k)\right](A-B)}{4} L_{1}(k) w^{2}(z)+\ldots
$$

Now from Lemma 3, equation (2.11) and equation (2.12), we have

$$
a_{2}=\frac{(A-B) L_{1}(k)}{2\{q+\beta[2, q]\} \varphi_{1}} c_{1}
$$

and

$$
a_{3}=\frac{(A-B) L_{1}(k)}{2[2, q]\{q+\beta[3, q]\} \varphi_{2}}\left\{c_{2}+\left\{\frac{2 D(k)-(1+B) L_{1}(k)}{2}+\frac{(A-B)}{2\{q+\beta[2, q]\}} L_{1}(k)\right\} c_{1}^{2}\right\} .
$$


Therefore

$$
\begin{aligned}
\left|a_{3}-\mu a_{2}^{2}\right|= & \frac{(A-B) L_{1}(k)}{2[2, q]\{q+\beta[3, q]\} \varphi_{2}} \mid c_{2}+\left\{\frac{2 D(k)-(1+B) L_{1}(k)}{2}\right. \\
& \left.+\frac{(A-B)}{2\{q+\beta[2, q]\}} L_{1}(k)\left(1-\mu \frac{\varphi_{2}}{\left(\varphi_{1}\right)^{2}}\right)\right\} c_{1}^{2} \mid
\end{aligned}
$$

This gives

$$
\begin{aligned}
\left|a_{3}-\mu a_{2}^{2}\right|= & \frac{(A-B) L_{1}(k)}{2[2, q]\{q+\beta[3, q]\} \varphi_{2}} \mid c_{2}-c_{1}^{2}+\left\{1+\frac{2 D(k)-(1+B) L_{1}(k)}{2}\right. \\
& \left.+\frac{(A-B)}{2\{q+\beta[2, q]\}} L_{1}(k)\left(1-\mu \frac{\varphi_{2}}{\left(\varphi_{1}\right)^{2}}\right)\right\} c_{1}^{2} \mid .
\end{aligned}
$$

Suppose that $\mu>\delta_{1}$, then using the estimate $\left|c_{2}-c_{1}^{2}\right| \leq 1$ from Lemma 3 and the well known estimate $\left|c_{1}\right| \leq 1$ of the Schwarz lemma, we obtain

$$
\begin{aligned}
\left|a_{3}-\mu a_{2}^{2}\right| \leq & \frac{(A-B) L_{1}(k)}{2[2, q]\{q+\beta[3, q]\} \varphi_{2}} \mid\left\{2+\frac{2 D(k)-(1+B) L_{1}(k)}{2}\right. \\
& -\frac{(A-B)}{2\{q+\beta[2, q]\}} L_{1}(k)\left(1-\mu \frac{\varphi_{2}}{\left(\varphi_{1}\right)^{2}}\right) \mid
\end{aligned}
$$

The inequality (2.15) is our required assertion (2.8) for $\mu>\delta_{1}$. On the other hand if $\mu<\delta_{2}$, then (2.13) gives

$$
\begin{aligned}
\left|a_{3}-\mu a_{2}^{2}\right| \leq & \frac{(A-B) L_{1}(k)}{2[2, q]\{q+\beta[3, q]\} \varphi_{2}}\left[\left|c_{2}\right|+\left\{\frac{2 D(k)-(1+B) T_{1}(k)}{2}\right.\right. \\
& \left.\left.+\frac{(A-B)}{2\{q+\beta[2, q]\}} L_{1}(k)\left(1-\mu \frac{\varphi_{2}}{\left(\varphi_{1}\right)^{2}}\right)\right\}\left|c_{1}\right|^{2}\right] .
\end{aligned}
$$

Applying the estimates $\left|c_{2}\right| \leq 1-\left|c_{1}\right|^{2}$ of Lemma 3 and $\left|c_{1}\right| \leq 1$, we have

$$
\begin{aligned}
\left|a_{3}-\mu a_{2}^{2}\right| \leq & \frac{(A-B) L_{1}(k)}{2[2, q]\{q+\beta[3, q]\} \varphi_{2}}\left[\left\{\frac{2 D(k)-(1+B) T_{1}(k)}{2}\right.\right. \\
& \left.\left.+\frac{(A-B)}{2\{q+\beta[2, q]\}} L_{1}(k)\left(1-\mu \frac{\varphi_{2}}{\left(\varphi_{1}\right)^{2}}\right)\right\}\right] .
\end{aligned}
$$

This is the last inequality in (2.8). Finally if $\delta_{1}<\mu<\delta_{2}$, then

$$
\left|\frac{2 D(k)-(1+B) L_{1}(k)}{2}+\frac{(A-B)}{2\{q+\beta[2, q]\}} L_{1}(k)\left(1-\mu \frac{\varphi_{2}}{\left(\varphi_{1}\right)^{2}}\right)\right| \leq 1 .
$$

Therefore (2.13), yields

$$
\begin{aligned}
\left|a_{3}-\mu a_{2}^{2}\right| & \leq \frac{(A-B) L_{1}(k)}{2[2, q]\{q+\beta[3, q]\} \varphi_{2}}\left\{\left|c_{2}\right|+\left|c_{1}\right|^{2}\right\} \\
& \leq \frac{(A-B) L_{1}(k)}{2[2, q]\{q+\beta[3, q]\} \varphi_{2}}\left\{1-\left|c_{1}\right|^{2}+\left|c_{1}\right|^{2}\right\}, \\
& \leq \frac{(A-B) L_{1}(k)}{2[2, q]\{q+\beta[3, q]\} \varphi_{2}} .
\end{aligned}
$$

We get the middle inequality in (2.8). This completes the proof. 
Theorem 2.4. Let $0 \leq k<\infty,-1 \leq B<A \leq 1$, be fixed and let $f(z) \in k-\mathcal{U S}_{q}(\lambda, A, B, \beta)$ and is of the form (1.1) Then for a complex number $\mu$.

$$
\left|a_{3}-\mu a_{2}^{2}\right| \leq \frac{(A-B) L_{1}(k)}{2[2, q]\{q+[3, q] \beta\} \varphi_{2}} \max \{1,|2 v-1|\}
$$

where $v$ is given by (2.17).

Proof. From (2.13) we have

$$
\begin{aligned}
\left|a_{3}-\mu a_{2}^{2}\right|= & \frac{(A-B) L_{1}(k)}{2[2, q]\{q+[3, q] \beta\} \varphi_{2}} \mid c_{2}-\left\{\frac{(1+B) L_{1}(k)-2 D(k)}{2}\right. \\
& \left.-\frac{(A-B)}{2\{q+\beta[2, q]\}} L_{1}(k)\left(1-\mu \frac{\varphi_{2}}{\left(\varphi_{1}\right)^{2}}\right)\right\} c_{1}^{2} \mid, \\
= & \frac{(A-B) L_{1}(k)}{2[2, q]\{q+[3, q] \beta\} \varphi_{2}}\left|c_{2}-v c_{1}^{2}\right|
\end{aligned}
$$

where

$$
v=\frac{(1+B) L_{1}(k)-2 D(k)}{2}-\frac{(A-B)}{2\{q+\beta[2, q]\}} L_{1}(k)\left(1-\mu \frac{\varphi_{2}}{\left(\varphi_{1}\right)^{2}}\right) .
$$

Applying the Lemma 2 on equation (2.16), we obtain the required result.

\section{REFERENCES}

[1] N. I. Ahiezer, Elements of theory of elliptic functions, Moscow, 1970.

[2] S. Hussain, S. Khan, M. A. Zaighum and M. Darus, Certain subclass of analytic functions related with conic domains and associated with Salagean q-differential operator, AIMS Math. 2(4)(2017), 622-634.

[3] S. Hussain, S. Khan, M. A. Zaighum, M. Darus and Z. Shareef, Coefficients Bounds for Certain Subclass of Biunivalent Functions Associated with Ruscheweyh q-Differential Operator, J. Complex Anal. 2017 (2017), Article ID 2826514.

[4] W. Janowski, Some extremal problems for certain families of analytic functions, Ann. Polon. Math. 28 (1973) $297-326$.

[5] S. Kanas, A. Wisniowska, Conic regions and k-uniform convexity, J. Comput. Appl. Math, 105 (1999), 327-336.

[6] S. Kanas, A. Wisniowska, Conic domains and starlike functions, Rev. Roumaine Math. Pures Appl. 45 (2000), $647-657$.

[7] S. Kanas, D. Raducanu, Some class of analytic functions related to conic domains, Math. slovaca, 64(5) (2014), 1183-1196.

[8] F. R. Keogh, E. P. Merkes, A coefficient inequality for certain classes of analytic functions, Proc. Amer. Math. Soc, 20 (1969), 8-12.

[9] N. Khan, B. Khan, Q. Z. Ahmad and S. Ahmad, Some Convolution Properties of Multivalent Analytic Functions, AIMS Math. 2 (2) (2017), 260-268.

[10] W. Ma, D. Minda, A unified treatment of some special classes of univalent functions. In: Proc. of the Conference on Complex Analysis (Tianjin), 1992 (Z. Li, F. Y. Ren, L. Yang, S. Y. Zhang, eds.), Conf. Proc. Lecture Notes Anal., Vol. 1, Int. Press, Massachusetts, 1994, 157-169.

[11] K. I. Noor, S. N. Malik, On coefficient inequalities of functions associated with conic domains, Comput. Math. Appl, 62(2011), 2209-2217.

[12] K. I. Noor, J. Sokol and Q. Z. Ahmad, Applications of conic type regions to subclasses of meromorphic univalent functions with respect to symmetric points, Rev. R. Acad. Cienc. Exactas Fs. Nat., Ser. A Mat. 111 (2017), 947C958. 
[13] K. I. Noor, J. Sokól and Q. Z. Ahmad, Applications of the diffierential operator to a class of meromorphic univalent functions. J. Egyptian Math. Soc. 24 (2) (2016), 181-186.

[14] M. Nunokawa, S. Hussain, N. Khan and Q. Z. Ahmad, A subclass of analytic functions related with conic domain, J. Clas. Anal. 9 (2016), 137-149.

[15] W. Rogosinski, On the coefficients of subordinate functions, Proc. Lond. Math. Soc, 48 (1943), 48-82.

[16] S. T. Ruscheweyh, New criteria for univalent functions, Proc. Amer. Math. Soc, 49 (1975), 109-115.

[17] H. Selverman, Univalent functions with negative coefficients, Proc. Amer. Math. Soc, 51 (1975), 109-116.

[18] S. Shams, S. R. Kulkarni, J. M. Jahangiri, Classes of uniformly starlike and convex functions, Int. J. Math. Math. Sci, 55 (2004) 2959-2961. 